evidence of poorer outcomes or more infections $^{6}$.

Attention should also focus on other strategies to reduce resistance. As McCormick et al. point out, the use of pneumococcal vaccines will reduce resistance to some extent because the vaccine serotype strains are among those with the highest proportion of resistance to both penicillin and macrolides. Influenza vaccination also reduces antibiotic use in healthy adults ${ }^{11}$. Finally, several recent studies have shown that improved hand hygiene in schools is associated with substantial reductions in rates of respiratory illness, the condition for which most antibiotics are prescribed $^{12}$. Improved hand hygiene also reduces rates of methicillin-resistant $S$. aureus in hospitals ${ }^{13}$.

Knowing the speed with which resistance is appearing, and knowing that decreases in antibiotic resistance occur more slowly than increases, we may not be able to "sponge away the writing on this stone" in the time allotted to us. It appears that McCormick et al. will have an opportunity to see whether or not their projections are indeed true.

1. McCormick, A.W. et al. Geographic diversity and temporal trends of antimicrobial resistance in Streptococcus pneumoniae in the United States. Nat. Med. 9, 424-430 (2003).

2. Mulholland, K. Strategies for the control of pneumococcal diseases. Vaccine 17, S79-S84 (1999).

3. Appelbaum, P.C. Antimicrobial resistance in Streptococcus pneumoniae: an overview. Clin. Infect. Dis. 15, 77-83 (1992).

4. Metlay, J.P. Update on community-acquired pneumonia: impact of antibiotic resistance on clinical outcomes. Curr. Opin. Infect. Dis. 15, 163-167 (2002).

5. Lonks, J.R. et al. Failure of macrolide antibiotic treatment in patients with bacteremia due to erythromycin-resistant Streptococcus pneumoniae. Clin. Infect. Dis. 35, 556-564 (2002).

6. Bronzwaer, S.L. et al. A European study on the relationship between antimicrobial use and antimicrobial resistance. Emerg. Infect. Dis. 8, 278-282 (2002)

7. Garcia-Rey, C et al. Pharmacoepidemiological analysis of provincial differences between consumption of macrolides and rates of erythromycin resistance among Streptococcus pyogenes isolates in Spain. J. Clin. Microbiol. 40, 2959-2963 (2002).

8. Zhanel, G.G. et al. Pharmacodynamic activity of fluoroquinolones against ciprofloxacin-resistant Streptococcus pneumoniae. J. Antmicrob. Chemother. 49, 807-812 (2002).

9. Soares, $\mathrm{S}$. et al. Evidence for the introduction of a multiresistant clone of serotype 6B Streptococcus pneumoniae from Spain to Iceland in the late 1980s. J. Infect. Dis. 168, 158-163 (1993).

10. Martin, J.M. et al. Erythromycin resistant group A streptococci in schoolchildren in Pittsburgh. $N$ Engl. J. Med. 346, 1200-1206 (2002).

11. Nichol, K.L. et al. Effectiveness of live, attenuated intranasal influenza virus vaccine in healthy, work ing adults: a randomized controlled trial. JAMA 282, 137-144 (1999).

12. Guinan, M. et al. The effect of a comprehensive handwashing program on absenteeism in elementary schools. Am. J. Infect. Control 30, 217-220 (2002).

13. Pittet, D. et al. Effectiveness of a hospital-wide programme to improve compliance with hand hy giene. Lancet 356, 1307-1312 (2000)

\section{Department of Microbiology,}

Toronto Medical Laboratories and the Mount

Sinai Hospital, University of Toronto,

Toronto, Ontario, Canada

E-mail:dlow@mtsinai.on.ca or

amcgeer@mtsinai.on.ca

\title{
Breaking away from the breast
}

The secreted glycoprotein netrin-1 gained its fame as a neuronal guidance molecule. Now this molecule has moved on to the mammary gland. Srinivasan et al. report that netrin- 1 acts as a sort of molecular glue for cells in buds at the growing tips of the gland (shown here as a whole mount stained with $\beta$-galactosidase, captured with light microscopy). These buds form during the equivalent of puberty in the mouse, growing as fast as $0.5 \mathrm{~mm}$ per day from the nipple down into a fat pad. A group of multipotent cells that strain forward at the tip of the bud express a netrin receptor, neogenin, report the investigators in the March issue of Developmental Cell. Nestled behind these cells at the tip is a separate layer of cells, which, the authors found, express netrin-1. Netrin$1-$ neogenin interactions seem to

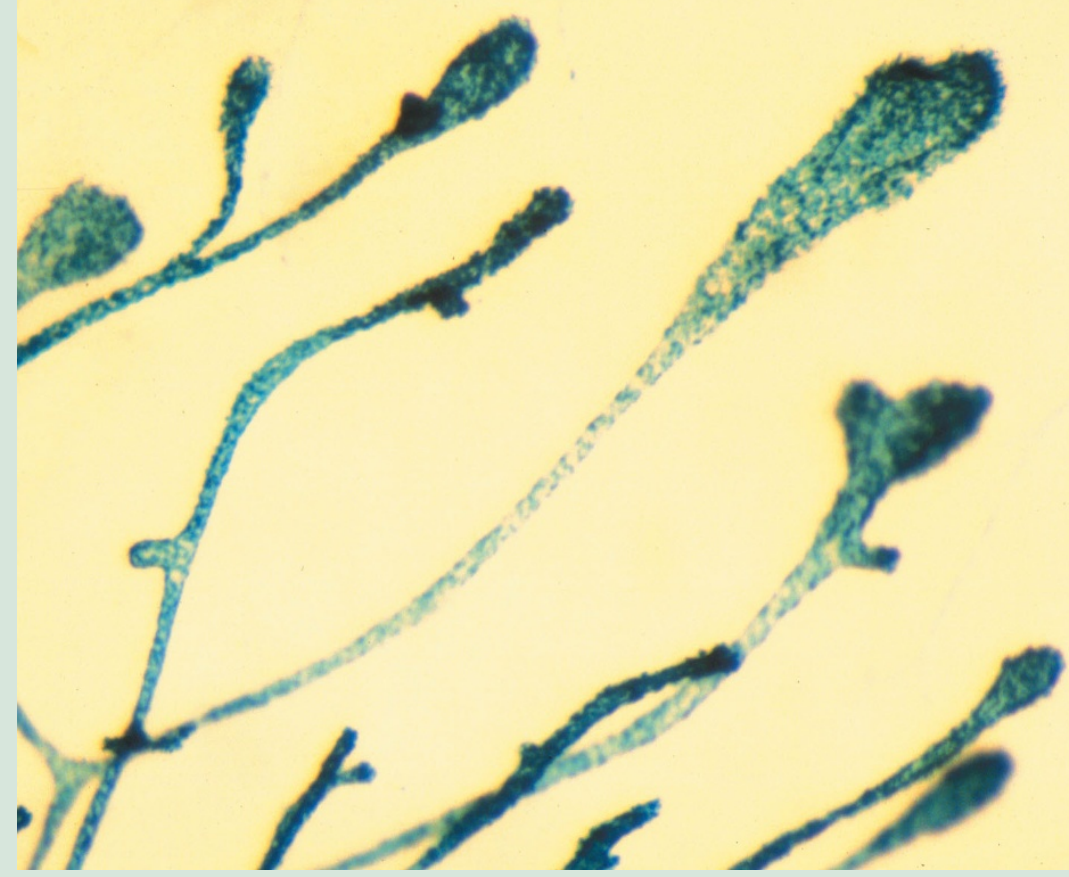
keep the two cell layers next to each other-and prevent the multipotent cells at the tip from wandering away. Such wandering occurs in glands lacking either netrin or neogenin, leading to cell death for some of the wayward cells. Developmental processes during adulthood may also involve netrin and neogenin, speculate the investigators, who found neogenin and netrin expressed during pregnancy and lactation. They are now taking a close look at whether loss of these genes leads to cancer susceptibility. Neogenin already has a well-known but inscrutable relative with cancer connections, DCC (deleted in colorectal carcinoma). 